\title{
RELAXATION TIMES IN STRICTLY DISK SYSTEMS
}

\author{
GEORGE B. RYBICKI
}

Smithsonian Astrophysical Observatory, Cambridge, Mass. 02138, U.S.A.

\begin{abstract}
It is shown that the time of relaxation by particle encounters of self-gravitating systems in the plane interacting by $1 / r^{2}$ forces is of the same order of magnitude as the mean orbit time. Therefore such a system does not have a Vlasov limit for large numbers of particles, unless appeal is made to some non-zero thickness of the disk. The relevance of this result to numerical experiments on galactic structure is discussed.
\end{abstract}

The use of the collisionless Boltzmann equation (Vlasov equation) for investigating galactic structure has been justified by simple estimates of the ratio of the relaxation time by particle encounters to the typical orbit time in the mean field (Chandrasekhar, 1939; Hénon, 1958; Ostriker and Davidsen, 1968). This ratio is of the order of $N / \log N$, where $N$ is the number of particles, so that for large $N$ the effects of particle encounters can be neglected on a mean field time scale.

A galaxy, even though it may be highly flattened, is still a three-dimensional system, and it is to three-dimensional systems that these estimates of relaxation time apply. However, galaxies are often approximated as strictly disk systems in which stars are still assumed to interact by $1 / r^{2}$ forces, but are constrained to move in a plane. Therefore, it is of some interest to consider the problem of relaxation times for strictly disk systems.

A simple order of magnitude estimate of relaxation time may be made as follows. For a system of $N$ particles, each of mass $m$, which is of typical size $R$, the virial theorem gives an estimate of a typical total particle velocity $V$ from the relation

$$
V^{2}=G N m / R,
$$

where $G$ is the gravitational constant. It is useful to distinguish between this total velocity and the typical random velocity $v$ of particles relative to a local frame of rest. This random velocity is some fraction of the total velocity:

$$
v=\lambda V, \quad 0<\lambda<1 \text {. }
$$

In a collision between two particles with relative velocity $v$ and with impact parameter $b$, the change of velocity is easily estimated to be

$$
\Delta v \sim G m /(b v) .
$$

This formula holds for $\Delta v \lesssim v$. It breaks down at the point where $\Delta v \gtrsim v$, which is the condition for a close encounter. This occurs when $b \lesssim b_{0}$, where

$$
b_{0}=G m / v^{2} \sim R /\left(\lambda^{2} N\right),
$$

using the virial theorem. 
The relaxation time $t_{R}$ may now be estimated as the time required for a typical particle to suffer a close encounter. Since this neglects the cumulative effect of longrange encounters, this will be an overestimate of the true relaxation time. In a time $t_{R}$ the motion of the particle takes it a distance $v t_{R}$ relative to neighboring particles. An estimate of the number of close encounters during this time is equal to the surface density of particles

$$
\varrho \sim N / R^{2}
$$

times the area $\left(v t_{R}\right) \cdot\left(2 b_{0}\right)$ within which another particle will cause a close encounter with the given particle. Setting this equal to unity leads to the result

$$
t_{R} \sim R^{2} /\left(2 N b_{0} v\right)
$$

The orbit time for a typical particle in the mean field is defined by

$$
t_{M}=R / V
$$

Combining these results yields

$$
t_{R} / t_{M} \sim \lambda / 2
$$

The relaxation time is seen to be at most the same order of magnitude as the mean orbit time, independent of the number of particles. Therefore the collisionless Boltzmann equation can never be an adequate description of a strictly disk system, however large.

A refined derivation will now be given that includes the cumulative effects of longrange encounters. In this case the relaxation time is defined as the time at which the root mean square velocity change due to encounters is of the same order of magnitude as the typical random velocity $v$. Thus, the condition is

$$
v^{2} \sim(\Delta v)_{\text {total }}^{2} \sim\left(G^{2} m^{2} / v^{2}\right)\left(v t_{R} \varrho\right) \int(2 \mathrm{~d} b) / b^{2} .
$$

The quantity $v t_{R} \varrho \cdot 2 \mathrm{~d} b$ represents the number of particle encounters during the time $t_{R}$ having impact parameters in the range $b$ to $b+\mathrm{d} b$. The lower limit of the integral is taken to be $b_{0}$ where the formula for $\Delta v$ breaks down and the divergence must be cut off. Since the integral converges rapidly for large $b$, the upper limit may be taken as $\infty$. Then

$$
t_{R} \sim v^{3} b_{0} /\left(2 G^{3} m \varrho\right)^{2} \sim v^{3} R^{3} /\left(2 G^{2} M^{2} N^{2} \lambda^{2}\right),
$$

where the results (4) and (5) have been used. With the virial theorem (1) and Equations (2) and (7) this gives

$$
t_{R} / t_{M} \sim \lambda / 2
$$

The fact that this estimate is identical with the previous one (8) indicates that the relaxation is substantially due to close encounters, and that the cumulative effect of long-range encounters is of no more than the same order of magnitude.

The relaxation time is seen to be proportional to $\lambda$, implying that the rate of relaxa- 
tion is greater when the system is cooler. However, as the system relaxes it heats up, decreasing the rate of relaxation; therefore a cool system still takes longer to reach any given stage of relaxation than a hotter one. If the parameter $\lambda$ is included in a similar three-dimensional derivation the relaxation time is proportional to $\lambda^{3}$.

Both of the above derivations suffer from the deficiency of assuming that the number of encounters in a certain impact parameter range may be computed simply on the basis of mean particle densities and typical relative velocities. The second derivation further assumes independence of encounters. The use of 'typical' velocities could be avoided by introducing velocity distribution functions, but we do not expect the order of magnitude estimates obtained here to be seriously altered. The assumption of independence of encounters does not present any difficulties in this two-dimensional case, since the relaxation is principally due to close encounters, and these encounters would be expected to occur independently. In this regard it is interesting to note that a long-range divergence occurs in the three-dimensional case when simple derivations of this sort are used. However this divergence does not occur here, and there is no need to introduce a long-range cutoff.

The use of mean particle densities implies lack of certain correlation effects, and this assumption would be difficult to validate rigorously. However, we offer the following rough argument: Because the relaxation is due to close encounters, in order for correlation effects to be serious there would have to be a severe alteration in the distribution of colliding particles having small impact parameters. Since the unperturbed orbits in the mean field are quite smooth this would require rather special synchronizations of the phases to avoid close encounters at orbit crossings. This seems unlikely in view of the great number of orbit crossings (order $N^{2}$ ) that must occur in a strictly plane geometry. Furthermore, it is difficult to imagine correlation effects producing the synchronizations necessary to avoid a close encounter when this is to occur at some great distance along the orbit.

It is of some interest that the difference between the two- and three-dimensional results may be traced to the different statistical weighting of impact parameters. In the three-dimensional case this is $2 \pi b \mathrm{~d} b$ while in the two-dimensional case it is $2 \mathrm{~d} b$. The extra factor of $b$ in the three-dimensional case tends to suppress the effect of close encounters and to weight the longer range encounters more. An interesting comparison between two- and three-dimensional systems is the average number of close encounters occurring in one mean field period. For two-dimensional systems it is of order $N$, while for three-dimensional system it is of order unity. This is the difference between each particle and one particle having a close encounter each mean field period.

These arguments concerning strictly disk systems of course do not apply to actual galaxies, which are three-dimensional; the validity of the Vlasov equation is well established in this case. However, strictly disk system approximations are commonly made in analytical and numerical treatments of galaxies, and it is necessary to judge these approximations in the light of the preceding results. For the analytical treatments there is no such problem of the relaxation time at all; the use of Vlasov theory is first established in view of the non-zero thickness of the disk, and then it is simply a 
question whether a two-dimensional form of the Vlasov equation is a good approximation to the three-dimensional form. Although this question is not trivial, at least it can be answered within the framework of Vlasov theory.

The situation regarding the numerical calculations (Miller and Prendergast, 1968; Miller et al., 1969; Hohl and Hockney, 1969), on the other hand, is not so straightforward. Here there is a strict zero thickness of the disk, and the relaxation results presented here apply. Therefore such calculations, if done sufficiently preciscly, are not faithful simulations of the Vlasov equation and thus do not apply to actual galaxies.

Fortunately, numerical calculations are themselves subject to further approximations that tend to reduce the severity of this difficulty. The grid size on which the potential (or force) is calculated effectively limits the close encounters that cause rapid relaxation. This discretization effect may be estimated as follows: Let $h$ be the distance over which the gravitational force is cut off by grid size effects or by some other effect, such as purposely altering the force law at short distances (Miller and Prendergast, 1968). Then a derivation of relaxation time may be given as before (see also Hohl, 1970), except that the lower limit on the integral in Equation (9) is taken to be $h$ rather than $b_{0}$.

This yields

$$
t_{R} / t_{M}=\lambda^{3} N h /(2 R)
$$

For a typical numerical experiment $N \sim 10^{5}$ and $h / R \sim 4 \times 10^{-2}$, so that $t_{R} / t_{M} \sim$ $\sim 2 \times 10^{3} \lambda^{3}$. For large values of random velocity $(\lambda \sim 1)$ the system might be followed for at least hundreds of mean periods, but for relatively 'cool' systems, say $\lambda \sim 0.1$ only several mean periods might be followed. For 'cold' systems $\lambda$ is zero, and relaxation would proceed rapidly, although the random velocities produced would then tend to stabilize the system against further rapid relaxation. By comparison a simple $N / \log N$ estimate would lead to an expectation of many mean periods of validity $\left(10^{3}-10^{4}\right)$, at least for relatively hot systems.

The numerical experiments of Hohl and Hockney (1969) were done with cold and relatively cool disks for up to five mean periods. The manner of breakup into condensations was interpreted by them in terms of the stability theory of Toomre (1964). It would seem, however, that the relaxation time effect described here might be operating as well, at least for the cold disks. An examination of the velocity distributions in these cases might be helpful in deciding whether the random velocities that developed were due to particle relaxation or mean field relaxation. Repeating the calculations with a mass spectrum might also be helpful. The experiments done with relatively cool disks, on the other hand, probably are not affected. This seems to be also in accord with recent calculations (Hohl, 1970).

The numerical experiments of Miller et al. (1969) are more difficult to assess, since their computations included a 'gas' component as well as a 'star' component. The gas component was artificially 'cooled' after each time step. One reason given for this cooling was that real gas clouds would be subject to the cooling mechanism of inelastic collisions. Aside from this physical reason they also state that such cooling is necessary 
to prevent rapid development of random velocities, which make it impossible to model a cool galaxy. However, in light of the discussion presented here one must question whether this latter presumed necessity for artificial cooling may simply be a result of the artificially rapid relaxation in strictly disk systems. Also the hot star populations which evolved in their calculations might be spurious for the same reason. Again, experimental checks can be done to answer these questions.

On the positive side, the estimates of relaxation time given here suggest some ideas for making numerical experiments more realistic and reliable. For example, a force law cutoff, such as used by Miller and Prendergast (1968) and Hohl (1970), would seem desirable in all such experiments. How much of a cutoff can be tolerated could be decided by suitable experimentation. In any case it is definitely clear that there is nothing to be gained by attempting to treat close encounters more exactly than has already been done.

Another way of minimizing the difficulty with relaxation time is to include, whenever possible, a fixed central force field, such as the galactic nucleus might produce (Hohl, 1970). This decreases the mean field time, leaving the relaxation time roughly unchanged; therefore more mean periods may be followed.

One experimental method of testing a computational procedure to see whether it is sensitive to the undesirable particle relaxation effects described here is to repeat a calculation using a mass spectrum. If the system is being correctly modeled as a Vlasov system there should be no effect; if not then there will be a tendency towards energy equipartition between the various masses.

\section{References}

Chandrasekhar, S.: 1939, Principles of Stellar Dynamics, Dover Publ., New York.

Hénon, M.: 1958, Ann. Astrophys. 21, 186.

Hohl, F. and Hockney, R. W.: 1969, J. Comp. Phys. 4, 306.

Hohl, F.: 1970, NASA TR R-343.

Miller, R. H. and Prendergast, K. H.: 1968, Astrophys. J. 151, 699.

Miller, R. H., Prendergast, K. H., and Quirk, W. J.: 1969, Univ. of Chicago Rept. COO-614-72.

Ostriker, J. P. and Davidsen, A. F.: 1968, Astrophys. J. 151, 679.

Toomre, A.: 1964, Astrophys. J. 139, 1217. 